Cahiers $d u$ MONDE RUSSE

\section{Cahiers du monde russe}

Russie - Empire russe - Union soviétique et États indépendants

$43 / 4 \mid 2002$

Intellectuels et intelligentsia

\title{
Nancy Shields Kollmann, By honor bound
}

\section{André Berelowitch}

\section{OpenEdition}

\section{Journals}

Édition électronique

URL : https://journals.openedition.org/monderusse/4010

DOI : $10.4000 /$ monderusse. 4010

ISSN : $1777-5388$

\section{Éditeur}

Éditions de l'EHESS

\section{Édition imprimée}

Date de publication : 30 décembre 2002

Pagination : 688-691

ISBN : 2-7132-1796-2

ISSN : $1252-6576$

Référence électronique

André Berelowitch, "Nancy Shields Kollmann, By honor bound ", Cahiers du monde russe [En ligne] 43/4 | 2002, mis en ligne le 17 juin 2009, consulté le 02 septembre 2022. URL : http://

journals.openedition.org/monderusse/4010; DOI : https://doi.org/10.4000/monderusse.4010

Ce document a été généré automatiquement le 2 septembre 2022

Tous droits réservés 


\title{
Nancy Shields Kollmann, By honor bound
}

\author{
André Berelowitch
}

\section{RÉFÉRENCE}

Nancy Shields KOLLMANN, By honor bound. State and society in early modern

Russia. Ithaca-Londres, Cornell University Press, 1999, 296 p.

1 L'honneur tient une place primordiale, en apparence du moins, dans les sociétés de l'Europe moderne, où l'on n'a que ce mot à la bouche, mais beaucoup plus modeste dans les préoccupations des historiens. Avant la parution de By honor bound, l'historiographie de l'honneur en Russie se résumait à quelques articles. Nancy Kollmann a donc écrit un livre neuf, dont le but n'est pas seulement d'éclaircir la nature de l'honneur moscovite, mais aussi de s'en servir comme d'un révélateur pour comprendre la société russe entre 1600 et 1800. Elle refuse en effet de considérer le règne de Pierre le Grand comme une césure et met plutôt l'accent sur la continuité du processus de modernisation entrepris par les souverains russes dès le milieu du XVII e siècle - point de vue que défend également Aleksandr Kamenskij ${ }^{1}$ (p. 212, 252).

2 L'ouvrage est sérieux et bien documenté, utile aux spécialistes comme aux nonspécialistes. La bibliographie, très copieuse, reflète les ambitions de l'auteur, qui entend situer le phénomène étudié dans un cadre théorique (d'où la place accordée à l'anthropologie et à la sociologie) et dans une perspective comparatiste. Utilisant les sources imprimées et les documents d'archives, elle a compilé un corpus comprenant plus de mille affaires de préséance et plus de six cents plaintes pour déshonneur.

3 L'introduction rappelle le contexte historique et présente les sources de la recherche. Puisque les Russes de cette époque commencent seulement à réfléchir sur eux-mêmes et n'ont donc pas produit de traité sur l'honneur, le premier chapitre cherche à reconstituer la manière dont ils le conçoivent, à partir de la législation et des valeurs qu'elle véhicule. La Justice russe (XI-XIII siècle) prévoit déjà des peines pour venger l'honneur des personnes 
insultées par des paroles ou par des actes. Ce n'est pas le duel, en effet, comme dans une grande partie de l'Europe occidentale, mais le procès qui permet à la victime d'obtenir satisfaction. Des amendes considérables, assorties au XVII siècle de prison ou de bastonnade, sont infligées par les juges. Ces amendes sont minutieusement tarifées, selon la nature de l'offense bien sûr, mais aussi selon les grades et conditions respectifs des parties (p. 49-58).

Le second chapitre traite principalement de l'honneur des femmes et de la protection, paradoxale selon l'auteur, que lui accorde une société patriarcale. Le déroulement des procès pour déshonneur est décrit dans le troisième, celui des conflits de préséance dans le quatrième chapitre. Le cinquième, le plus réussi du livre, se propose de situer la défense de l'honneur parmi les différentes stratégies d'intégration sociale mises en œuvre par la monarchie russe. Aux XVIII et $\mathrm{xIX}^{\mathrm{e}}$ siècles, conception de l'honneur et législation se rapprochent peu à peu de celles qui ont alors cours en Europe occidentale (chapitre VI, épilogue).

5 L'analyse du corpus révèle que les insultes mettent le plus souvent en cause l'honnêteté du clan adverse ou la vertu de ses femmes (p. 46-47); que les deux tiers des procès pour raison d'honneur opposent des personnes de rang sensiblement égal (p. 96-97); que plus de la moitié des plaintes sont retirées avant le début du procès, pour régler l'affaire à l'amiable (p.115-116); que les juges se conforment, dans l'ensemble, aux prescriptions des codes en vigueur (p.122-123, 189-191). Malheureusement, après avoir vanté le caractère concret et vivant de ses sources, l'auteur ne nous en donne, à de rares exceptions près, que de brefs résumés ou des typologies superficielles. Les quelques détails cités ne font qu'aviver les regrets du lecteur : plainte, en 1639-1640, d'indigènes sibériens « déshonorés » par le gouverneur russe local, qui leur offre de la bière au lieu d'eau de vie (Nancy Kollmann traduit, à tort, vino par wine) ; plainte des Otrep'ev en 1671, perdus de réputation par la faute de leur parent Grégoire (le premier Faux Dimitri du Temps des Troubles) (p. 51-52).

6 Dispersés entre plusieurs chapitres, les résultats de l'enquête n'occupent qu'une faible partie du livre, l'essentiel, aux yeux de l'auteur, étant d'établir un lien entre les particularités du sentiment russe de l'honneur et la structure de la société. En décrivant celle-ci, Nancy Kollmann réfute, à juste titre mais sans toujours fournir de démonstration, une série de stéréotypes qui ont la vie dure, puisque certains remontent à Herberstein ou Olearius.

7 La monarchie moscovite n'est pas un despotisme (p. 8, 17-20), non seulement parce que les contraintes de l'espace, du climat, de la démographie l'obligent à la souplesse, mais aussi parce qu'elle gouverne en s'appuyant sur le consensus des sujets : « L'autocratie ne cherchait pas à isoler le monarque et ses hommes dans la sphère autonome du pouvoir, mais à faire participer l'ensemble de la société à l'exercice de ce pouvoir» (p. 202). D'où le rôle important des communautés locales (p. 15, 95, 112-113), la consultation, fréquente, des bojare (conseillers du prince), plus épisodique, des assemblées d'états (p.177-180, 200-201). Ce fonctionnement élastique du pouvoir tendrait à rapprocher la Russie d'une Europe occidentale que nous commençons à mieux connaître derrière la façade imposante des institutions (p. 62, 107).

8 Les différences sont ailleurs, dans la domination exclusive du religieux (c'est la Goddependant society de D. Rowland) et dans le style extensif du développement, dont Nancy Kollmann suppose qu'il a été " préféré » par les gouvernants russes (p. 180). Je ne crois pas qu'ils aient réellement eu le choix. 
9 Le ciment de cette société consensuelle est l'honneur, non pas l'amour-propre vétilleux du gentilhomme castillan ou français, mais une variante profane du sentiment religieux. $\mathrm{Au}$ sommet du système, le tsar, oint du Seigneur, détenteur des regalia, arbitre et distributeur de l'honneur et des dignités. De cette source inépuisable, l'honneur s'écoule en cascade vers la base de l'édifice social, imprégnant au passage l'espace sacré du Kremlin, les lieux de pélerinage des tsars, les objets dont ils se servent et jusqu'aux monnaies qu'ils font frapper. Les agents qui les représentent en sont également investis ; les chaînes de commandement servent à transmettre les ordres, mais aussi le mana de proche en proche jusqu'aux plus humbles des administrés. Ainsi s'explique la protection contre le déshonneur accordée à toutes les conditions sociales, serfs, mendiants, jongleurs, devineresses et prostituées compris, comme à toutes les confessions (p. 47, 50).

10 Nancy Kollmann ne met pas suffisamment en valeur ces pages (187-199), qui sont les plus fortes du livre et contiennent la véritable réponse au problème qu'elle s'est posé. Son hypothèse mérite d'être approfondie et vérifiée.

11 Les mérites réels de l'ouvrage ne doivent cependant pas faire oublier ses non moins réels défauts. L'auteur manque de rigueur dans la traduction des sources : rozveden ne signifie pas que l'un des protagonistes d'une querelle de préséance est « considéré comme égal » à son adversaire (p.157), mais que sa nomination a été annulée, presque toujours parce qu'il est inférieur à celui-ci (Dvorcovye Razrjady, I, 410, 437). Polotskij n'est pas un nom de famille: Siméon est de Polotsk comme Jean est de Meung. Vor, vorovstvo, traduits par thief, thievery, ne signifient jamais "voleur, larcin» (qui se disent tat', tat'ba), mais désignent la malhonnêteté, depuis la simple tricherie jusqu'au banditisme et à l'imposture (Tušinskij vor). L'expression vydača golovoj est rendue par surrender by the head, qui ne veut strictement rien dire. Golova est ici l'équivalent de la vie, et l'instrumental ne signifie pas "au moyen de », mais " jusqu'à », "y compris». Il ne s'agit pas là d'un détail, puisque la reddition à merci du perdant dans un procès pour déshonneur ou un conflit de préséance est le seul fil qui rattache les procédures moscovites au duel occidental (cf. Cahiers du Monde russe et soviétique, 34, 1-2, 1993, p. 130-131).

On relève des erreurs de fait et des interprétations discutables. L'auteur ne met pas en doute la réclusion des femmes (p. 82-84), dont Nada Boškowskaa démontré le caractère mythique $^{2}$. Lorsque la preuve judiciaire doit être fournie par un serment sur la croix, Nancy Kollmann écrit que le tirage au sort désigne celui des plaideurs «qui jurera le premier» (p.120). Mais il était impensable, pour les Russes de cette époque, de contraindre les deux parties au serment: l'une d'entre elles au moins se serait parjurée, mettant en danger son salut éternel (cf. Forschungen zur osteuropäischen Geschichte, 56, p. 20). Le dernier quart du XVII e siècle est traité de façon superficielle. Un certain nombre de mesures hétérogènes sont rapportées pêle-mêle à la volonté de modernisation, qui est indiscutable mais coexiste avec d'autres objectifs, comme la défense de l'orthodoxie (persécution des Vieux-Croyants) ou la remise en ordre de la Cour (p. 216-219).

Nancy Kollmann affirme, sans vérifier, qu'il n'existe hors de Russie rien de semblable à la lutte pour les places, ou mestničestvo (p.132); en réalité, même si les règles du jeu sont différentes, les conflits de préséance se ressemblent beaucoup, quels que soient le pays et l'époque. Des comparaisons précises permettent justement de mieux savoir en quoi réside l'originalité du système russe. Les injures ne sont pas « une stratégie visant à faire respecter les normes sociales» (p. 84), mais un défi lancé au clan adverse. Les lectures extensives de l'auteur (Péristiany, Pitt-Rivers...) auraient pu lui suggérer une analogie 
entre le schéma classique de l'affaire d'honneur (défi, contre-défi, réparation) et les conflits qu'elle a étudiés (cf. Cahiers du Monde russe et soviétique, 34, 1-2, 1993, pp. 126-127).

L'aspect le plus contestable du livre est le cadre théorique, que Nancy Kollmann emprunte à la sociologie mais ne songe pas à remettre en cause. C'est un fonctionnalisme radical : toute institution, toute pratique collective ont pour effet, et pour raison d'être, de perpétuer l'ordre social existant - ce qui est vrai, comme chacun sait, de tout groupe humain. À l'aide de cette doctrine tautologique, il devient facile d'expliquer, par les besoins de la société, le gouvernement consensuel et les querelles de préséance, la protection de l'honneur et la morale patriarcale. Au sujet de cette dernière, l'auteur s'interroge : un « code social plus égalitaire » n'aurait-il pas été plus efficace? Mais c'est pour se débarrasser aussitôt du problème: "Le patriarcat existait en tant que code culturel, affirmant le sentiment masculin de supériorité » (p. 71-72).

Il faut regretter que cet hégélianisme sans la dialectique ( Tout ce qui est réel est rationnel »), dérive prévisible de la cultural history américaine, empêche Nancy Kollmann de se poser certaines questions. Comment se fait-il, par exemple, que malgré toutes ces pratiques censées maintenir l'ordre social, le $\mathrm{xvII}^{\mathrm{e}}$ siècle ait été une suite presque ininterrompue de révoltes, débouchant sur une crise profonde que Pierre le Grand a eu tant de peine à résoudre - s'il l'a résolue ? Le principal défaut de la pensée toute faite, des formules toutes faites (status consciousness, cultural construct, manipulation of dominant discourses et autres virtutes dormitive) est de pousser au raisonnement circulaire.

On ne peut pas demander à une historienne de renoncer à ses convictions, mais il est permis de souhaiter que, dans les livres à venir, ces considérations abstraites tiennent moins de place et ne nous empêchent pas d'apprécier à leur juste valeur le travail acharné de Nancy Kollmann, son sens aigu du document et ses authentiques intuitions.

\section{NOTES}

1. A. B. Kamenskij, Ot Petra I do Pavla I (De Pierre I Ir $^{e}$ Paul I ${ }^{e r}$ ), Moscou, 1999, 576 p.

2. N. Boškowska, Die russische Frau im 17. Jh., Cologne-Weimar-Vienne, Böhlau Verlag, 1998, $497 \mathrm{p}$. 